19or) one can say that the scar of the tracheotomy wound is all that eminds one of his little adventure.

The interesting points of the case are to my mind the following: It is well known that in children paralysis of the adductors takes place very suddenly from slight causes, and, in face of this fact, I was disposed to question whether a portion of the nut had gone into a bronchus. Again, what was the position of the child as to "cold," and possibly false croup antecedent to the aocident? This could only be answered by the friends, and, as the boy had once before had false croup, a cold might easily have been overlooked if not severe. His general condition, also, was in favour of croup. These points must pass through one's mind when considering the treatment, and in this case they were duly balanced. The only direct evidence was that of the boy himself, that he had cracked and tried to eat the nut. 'The sudden oncoming of the attack favoured the idea of a foreign body, and the treatment adopted was subsequently proved correct by the pieces of husk found on the dressing.

I consider the success of the case due in great measure to the excellent nursing the boy received.

\section{A NOTE ON}

\section{ACUTE DILATATION OF THE HEART.}

BY H. OLIPHANT NICHOLSON, M.B., C.M.ABERD., Physician, New Town Dispensary, Edinburgh.

THE remarks which Dr. Lees makes on the accentuation of the second aortic sound in his admirable paper in the British Medical Journal of January $5^{\text {th }}$ on acute cardiac dilatation are of special interest to those who have employed graphic methods in the examination of the heart and pulse in this condition.

It is difficult to conceive at first sight how an accentuated aortic second sound can be produced with a general relaxation of the vessels and the feeble contraction of a degenerated ventricle, for the two principal factors concerned in the production of this sound are obviously at fault. Dr. Lees recognises that increased tension in the aorta is necessary for the production of this symptom, and seeks to explain it by suggesting that the arterioles of the splanchnic area are constricted.

Without denying that certain sections of the arterial system may contract or dilate in response to the irritation of toxins in the blood stream, and that under such circumstances the radial pulse may be unreliable as a true index of the blood pressure, still I do not imagine that any such explanation is required. Nor do I consider that the ingenious suggestions put forward by Dr. Biernacki are necessary to explain the symptom, though it would not seem improbable that, after the completion of the ventricular systole, the withdrawal. of the support of the contracted muscle from the root of the aorta may be more sudden than in health, and tend to set up a negative wave of greater velocity towards the aortic cusps. It seems to me that Dr. Lees has drawn attention to a clinical sign of very great interest and importance, the value of which may become more apparent should the explanation I now advance be accepted as the correct interpretation of the symptom.

Dr. Lees rightly assumes that there is increased tension in the aorta to account for this sign, but one cannot accept his explanation as the most probable one. I believe that in extreme dilatation of the heart the arterial blood pressure must always be relatively high. That is to say, if one considers the whole circulatory apparatus in this condition, and recognises the important fact that the ventricle is acting at its highest possible limit and exerting all its reserve power to maintain a pressure compatible with life, then one will understand that a radial artery in which the tension feels poor, which gives a tracing of so-called low tension with the sphygmograph, or a low reading in millimetres of mercury on the sphygmometer, may mean very little. The blood pressure is really relatively high for the state of the heart and blood vessels, and the true value of the accentuated aortic second sound would seem to be its occurrence at a time when, to judge from collateral symptoms, one would least of all expect it. Correctly interpreted, I imagine it may indicate that the ventricle is straining every sinew and that the heart is arriving at a stage where no further response to stimulation is possible. A little mone work is suddenly demanded and syncope oocurs. Therefore, marked accentuation of this sound may be classed with vomiting as a dange: signal of grave significance, and its persistence will lead one to exercise extreme watchfulness.

Numerous observations made with the sphygmograph and the sphygmometer in the cardiac dilatation of acute pneumonia in children, ${ }^{2}$ in rheumatism, and in many other conditions of circulatory failure, have led me to the conclusion that the blood pressure may be very much under the normal average stated by $\mathrm{Hill}^{2}$, namely, $100-110 \mathrm{~mm}$. Hg. in the re cumbent posture, and may yet be relatively high for the conditions existing at the time.

But as a matter of fact, in certain grave states of cardiac dilatation, and in advanced valvular disease, the actual blood pressure as tested by Hill's instrument may be enormously high. This fact impressed me very much, and can only be accounted for, I think, by admitting the explanation I have already given, namely, that the ventricle is called upon to work at its highest pressure. In observations made on a series of such cases a blood pressure of over $200 \mathrm{~mm}$. of $\mathbf{H g}$. was sometimes demonstrated, and the sphygmographs were occasionally markedly dicrotic, and in other ways suggestive of a low arterial tension. The coexistence of a typically dicrotic pulse wave with a normal blood pressure is recog. nised by Roy and Adami, ${ }^{3}$ who consider the nature of the heart's action a most important factor in the production of dicrotism. This statement finds confirmation in the fact that in the rapidly dilating heart of pneumonia and rheumatism in children the most significant and striking symptom is often the disappearance or great diminution of the dicrotic wave. A case of extreme rheumatic dilatation in a young child showed this feature when first examined, and there was also epigastric pulsation. The cardiograph proved this to be due to the greatly distended left ventricle and not to the right as is generally the case in dilatation of the adult heart. After a few days' rest in bed recovery was indicated by the reappearance of the dicrotic wave in the pulse curve and by the disappearance of epigastric pulsation.

Unfortunately I have not paid special attention to the accentuation of the second aortic sound in any of these cases, but the coexistence of advanced cardiac dilatation, feeblelowtension pulse, and immensely high systemic blood pressurewhich, I have repeatedly observed, certainly suggest that this sign would have been present. But the demonstration by the sphygmometer of an abnormally low pressure in the arteries as is usually found in the acute pneumonias of children, and which, as Dr. Biernacki has shown, occurs in diphtheria, is not, in my opinion, incompatible with the existence of an accentuated aortic second sound. On the contrary, the presence of this sign under such circumstances is full of meaning, and suggests that some qualification of the term "blood pressure" is very desirable, as what seems a high tension in the vessels at one time may really be a relatively low one and vice vers $\hat{a}$ when all the factors concerned in its maintainance are fully examined.

References.
1 British Medical Journal, September ist, roo. 2 Proc. Physiol. Soc. January 15th, 1898. 3 Practitioner, vol. ii, 1900, p. 23

\section{A NOTE ON THE TREATMENT OF GENU VALGUM.}

By E. MUIRHEAD LITTLE, F.R.C.S.ENG., Surgeon to the National Orthopædic Hospital.

In the treatment of slight cases of knock-knee in children in which osteotomy is not advisable, either splints or steel instruments are commonly used. I have found the arrangement of splints and the pocket on the boot illustrated herewith of great use in such cases. It is well known that the deformity disappears on flexion, and consequently it is only when the knee is fully extended that mechanical treatment can be effective. As may be seen in the figure, the necessary extension is maintained by means of a ham splint, which is fastened by its outer edge to the hinder edge of the long out side splint with two leather hinges. 